\title{
Historia vegetal y regímenes de fuego recientes de la turbera costera de Chepu, Isla Grande de Chiloé, Chile
}

\section{Recent plant history and fire regimes of the coastal peat bog of Chepu, Great Island of Chiloé, Chile}

\author{
J. Max Troncoso Castro ${ }^{1 *}$, Alfredo Saldaña ${ }^{2} \&$ Mauricio J. Rondanelli-Reyes ${ }^{1}$ \\ 'Laboratorio de Palinología y Ecología Vegetal, Departamento de Ciencia y Tecnología Vegetal, Universidad de Concepción, \\ Campus Los Ángeles, Chile. \\ 2Departamento de Botánica, Universidad de Concepción, Casilla 160-C, Concepción, Chile. \\ *josetroncoso@udec.cl
}

\section{RESUMEN}

La reconstrucción de la vegetación a través del análisis polínico y los regímenes de incendio inferidos a partir de partículas de carbón son de gran importancia para el estudio de fenómenos climáticos pasados, tales como la Pequeña Edad de Hielo y el Periodo Cálido Medieval. El objetivo de este estudio es reconstruir la historia vegetacional de la turbera "Chepu", de origen antrópico, ubicada en la Isla Grande de Chiloé, Región de Los Lagos, Chile, del último milenio, a través del análisis palinológico y del análisis de alta resolución de partículas de carbón macroscópico. Para esto se realizó un sondaje estratigráfico en el centro de la turbera con el fin de proceder al análisis de polen, obtener dataciones y cuantificar las partículas de carbón macroscópicas. El modelo de edad se obtuvo a partir de tres dataciones radiométricas y $\mathrm{PB}^{210}$ utilizando el programa MCAge. Los resultados obtenidos señalan que los patrones de fuego han estado supeditados principalmente a las condiciones climáticas reinantes en el área, observándose en la mayoría de los casos concordancia entre eventos de fuego locales y extralocales en la zona en estudio. Este régimen de fuego tuvo influencia sobre la abundancia de especies, denotándose una disminución del componente arbóreo en períodos de actividad de fuego. Se observan a través del diagrama polínico cambios en la vegetación y en la actividad de fuego durante los últimos 2.000 años, asociados principalmente a variaciones en las precipitaciones y a la probable acción del hombre durante el siglo XX, respectivamente. Entre 1960 y 1961 años cal. AD se observa en el perfil un cambio en la composición y abundancia de los taxa vegetales, asociado al evento del megaterremoto de 1960, en Chile. Los datos aportados por este perfil son aún insuficientes para establecer la influencia climática extralocal de eventos tales como la Pequeña Edad de Hielo o el Período Cálido Medieval, en Chiloé; aunque se observan ciertas tendencias.

Palabras clave: Historia vegetacional, regímenes de fuego, Isla Grande de Chiloé, polen, carbón.

\begin{abstract}
The reconstruction of vegetation through pollen analysis and the fire regimes inferred from charcoal particles are of great importance to the study of past climatic phenomena such as the Little Ice Age and the Medieval Warm Period. The aim of this study was to reconstruct the vegetational history of the "Chepu" anthropic peat bog, located on the Great Island of Chiloé in the Los Lagos Region of Chile, of the last millennium through palynological analysis and a high-resolution analysis of macroscopic charcoal particles. This was done doing a stratigraphic boring in the center of the bog to conduct the pollen analysis, obtain dating and quantify the macroscopic charcoal particles. The age model was obtained from three radiometric datings and $\mathrm{PB}^{210}$ using the program MCAge. The results indicate that the fire patterns have been mainly contingent upon the prevailing climatic conditions in the area, with consistency being observed in most cases between local and extralocal fire events in the study area. This fire regime has influenced the abundance of species, indicating a decrease of the arboreal component in periods of fire activity. Changes in the vegetation and fire activity are observed through the pollen diagram of the last 2,000 years, associated mainly with variations in precipitation and likely with human activity during the $20^{\text {th }}$ century, respectively. Between 1960 and 1961 cal. AD in the profile, a change was observed in the composition and abundance of plant taxa associated with the megathrust earthquake in Chile in 1960. The data contributed by this profile are still insufficient to establish the extralocal climatic influence of events such as the Little Ice Age or the Medieval Warm Period on Chiloé, but certain tendencies are observed.
\end{abstract}

KeYwords: Vegetational history, fire regimes, Great Island of Chiloé, pollen, charcoal. 


\section{INTRODUCCIÓN}

Las investigaciones palinológicas en la región sur de Sudamérica han hecho hincapié en la importancia del cambio climático, las migraciones y las quemas que han contribuido al cambio de la vegetación a gran escala durante el Cuaternario tardío (Heusser 1987, Markgraf 1989, Villagrán et al. 1996, Moreno et al. 2010, 2012), pero la importancia de las perturbaciones naturales en escalas de tiempo de siglos han sido poco investigadas; son escasos los estudios paleoecológicos y paleoclimáticos de alta resolución temporal que permitan dilucidar las interrelaciones existentes entre el clima, la vegetación y el régimen de incendios durante el Holoceno. En este contexto, en Chile, la Isla Grande de Chiloé es una de las zonas que muestra los mayores cambios en el paisaje durante esta época (Abarzúa et al. 2004, Abarzúa \& Moreno 2008).

El Holoceno ( los últimos 11.500 años) ha sido caracterizado a nivel global como un periodo de mucha variabilidad climática a escalas de tiempo de milenios, centurias y décadas. Durante el Holoceno se desarrollaron las sociedades humanas y la agricultura, las cuales se vieron afectadas o modeladas por esta variabilidad ambiental natural (Moreno et al. 2009a). Durante estos ciclos de cambios, la temperatura y la precipitación han sido señaladas, para el sur de Sudamérica, como variables determinantes en la estructuración del paisaje, específicamente para la región de la Patagonia, a través de diferentes análisis de datos en testigos obtenidos de turberas y lagos (Heusser 1995, Markgraf et al. 2003, Huber et al. 2004, Wille et al. 2007, Wille \& Schäbitz 2009, Moreno et al. 2009b, Rondanelli et al. 2011).

Los ecosistemas de turbera son ideales para estudiar los cambios ambientales ocurridos en el pasado, dado que los sedimentos depositados registran las características del ambiente de la turbera y sus alrededores al momento de su depositación (Iturraspe \& Roig 2000). El estado del conocimiento de las turberas chilenas es en general deficiente (Schlatter \& Schlatter 2004). Algunos estudios puntuales han establecido la dinámica de sucesión de especies leñosas en turberas de Sphagnum (Díaz 2004), el impacto de la explotación comercial del Sphagnum (Zegers et al. 2006) y la determinación en la distribución espacial de plantas vasculares sobre Sphagnum (Teneb \& Dollenz 2004).

En el límite norte de la Patagonia occidental de Chile se ubica geográficamente la Isla Grande de Chiloé, la cual posee grandes extensiones de turberas. Las turberas naturales de Chiloé tienen acumulaciones de turba superiores a $1 \mathrm{~m}$ de profundidad y datan desde el último ciclo glacial (18 a 20 mil años AP), cuando ocuparon gran parte del territorio de Chiloé (Villagrán 2001). Las turberas antropogénicas presentan menor acumulación de turba y se forman sobre el suelo mineral después de incendios de bosques o grandes talas rasas que datan desde fines del siglo XIX. Muchos de estos pomponales se han transformado en un tipo de frontera agrícola-forestal (Jaña-Prado et al. 2006), donde el proceso de cambio de uso del suelo está ocurriendo con gran rapidez (Echeverría et al. 2007), asociado a la fragmentación y conversión de los bosques nativos en otros tipos de cobertura antropogénica tales como praderas, plantaciones de eucaliptos, turberas antropogénicas de Sphagnum (pomponales), y matorrales (Carmona et al. 2010). Por lo tanto, una turbera antropogénica resulta ser un laboratorio natural propicio para el estudio de la variabilidad ambiental temporal, de alta resolución.

La presente investigación realizada en la Isla Grande de Chiloé, Región de Los Lagos, se focalizó en el análisis polínico de una turbera antropogénica localizada en el sector de "Chepu", comuna de Ancud, en el extremo nor-oeste de Chiloé. El objetivo general del estudio fue reconstruir la historia vegetacional de este pomponal a través del último milenio, utilizando para ello el análisis de polen y de carbón macroscópico con el fin de establecer si es posible, sobre la base de esta historia, reconocer la influencia de alguna anomalía climática zonal o azonal, así como la influencia del fuego en el modelado de la vegetación de este ecosistema.

\section{MATERIALES Y MÉTODOS}

Área De ESTUdio

La turbera Chepu (4203'10,8'S; 7359'56,8”W) (Fig. 1) se encuentra a una altitud de $5 \mathrm{~m}$ s.n.m. y a una distancia de la costa de 2,59 km, se ubica en la cuenca del río Chepu en la primera cuenca hidrográfica importante que desagua hacia el Océano Pacífico en el norte de la Isla Grande de Chiloé, constituida por numerosos afluentes (Puntra, Butalcura, Coluco, Metalqui, entre otros) y disecta los relieves de baja montaña situados al poniente, donde predomina el bosque pluvial siempre verde (Quintanilla 2004).

Aproximadamente unos 8-10 $\mathrm{km}$ antes de la desembocadura del Chepu, las riberas del río están inundadas con bosquetes que se desarrollan sobre el agua, como consecuencia del hundimiento de poco más de un metro del terreno producto del terremoto del año 1960 (Godley \& Moar 1973).

El área septentrional de la Isla Grande de Chiloé, donde se localiza la cuenca del río Chepu, posee características de un clima templado lluvioso con precipitaciones anuales de alrededor de $2.600 \mathrm{~mm}$ y con una disminución importante en los meses de verano. Las temperaturas máximas llegan a poco más de los $14^{\circ} \mathrm{C}$ y las mínimas medias anuales alcanzan a los $10^{\circ} \mathrm{C}$ (Di Castri \& Hajek 1976). En los lugares bajos predomina formación de turba donde se desarrolla mejor Tepualia stipularis (Hook. et Arn.) Griseb. y cuando el horizonte A posee mayor presencia de arcilla y humus, alcanzan un mejor desarrollo las Mirtáceas y Nothofagus. 
En general, los suelos presentan elevados contenidos de materia orgánica entre los 0 y $25 \mathrm{~cm}$ aproximadamente (Donoso et al. 1984). En este sector, durante comienzos del siglo pasado, el ecosistema forestal estuvo constituido por el denominado tipo de bosque valdiviano pluvial (Donoso et al. 1984, 1993, Armesto et al. 1996). En los últimos 25-30 años la composición del bosque insular ha cambiado considerablemente debido a la roturación de ecosistemas para ganadería lechera y por la expansión de las superficies afectadas por incendios vegetales, desde el sector sur de la Isla y hacia el eje carretero Ancud-Quellón (Quintanilla 2004).
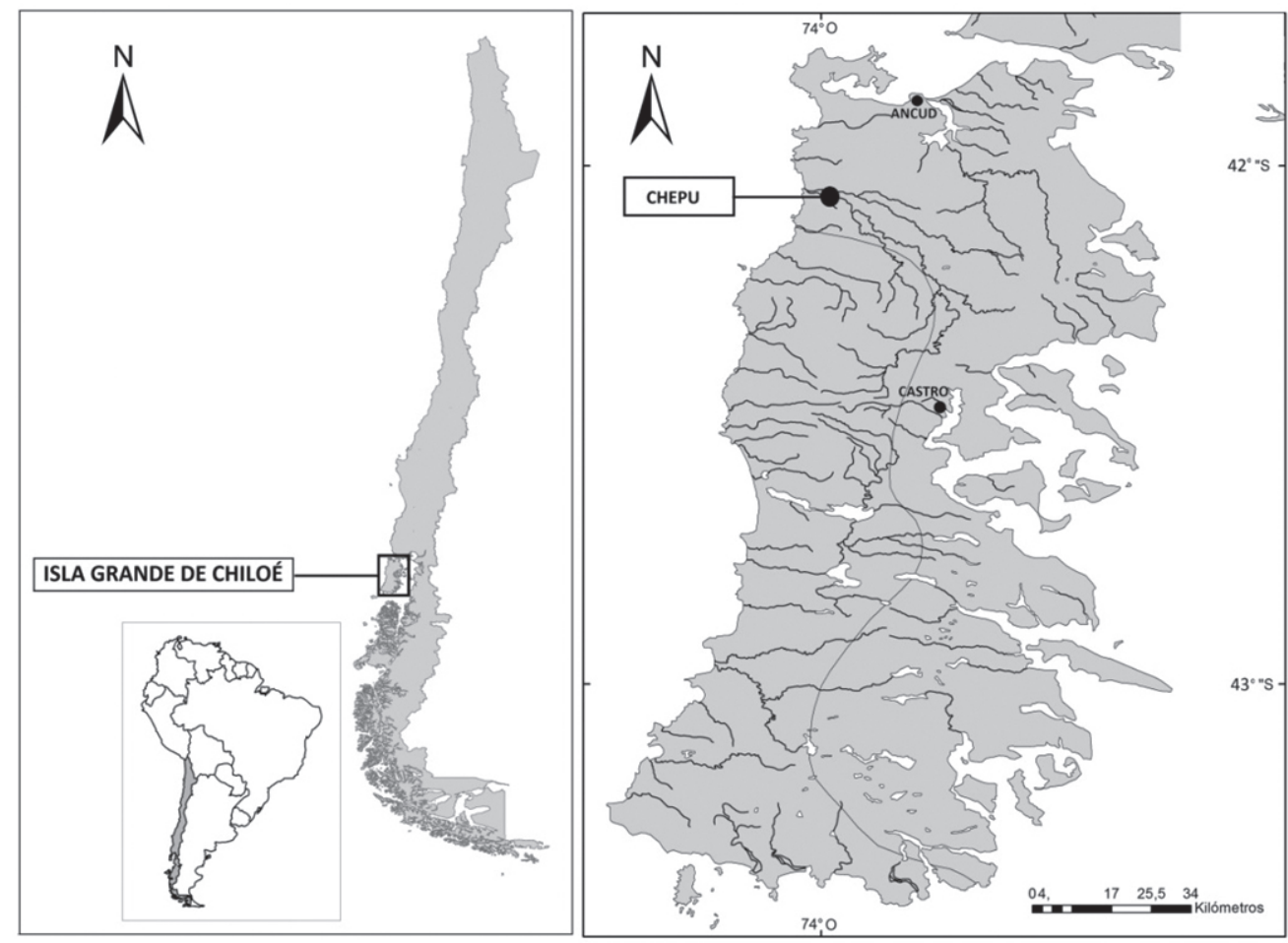

Figura 1. Mapa de ubicación de la Turbera Chepu.

Figure 1. Location map of the Chepu Peat Bog.

SONDAJE Y ANÁLISIS DE LABORATORIO

Para obtener el perfil estratigráfico se realizó un sondaje de $20 \mathrm{~cm}$ de profundidad en el centro de la turbera, con ayuda de una sonda Livingstone. Se analizó la totalidad del perfil, analizando los sedimentos cada $2,5 \mathrm{~cm}$, de base a techo. Las muestras fueron procesadas, para análisis palinológico, bajo la metodología clásica de Faegri e Iversen (1989). El recuento polínico alcanzó como mínimo 300 granos de polen por preparación, siguiendo el método de área mínima utilizado en los estudios de comunidades vegetacionales, adaptado al recuento polínico por Bianchi \& D’Antoni (1986). El recuento de micropartículas de carbón, presentes en las muestras, fue considerado como un taxón más dentro de la suma polínica, contabilizando sólo aquellas partículas cuyo tamaño sea igual o superior a $50 \mu \mathrm{m}$. El análisis de carbón vegetal macroscópico se llevó a cabo para reconstruir la historia local de incendios a través de los métodos de Whitlock \& Larsen (2001), tomando de forma continua y contigua $1 \mathrm{~cm}^{3}$ de material sedimentario a partir de cada centímetro de longitud del testigo. Los resultados de los conteos de partículas de carbón fueron convertidos a concentración de carbón (partículas de carbón/cc) y luego divididos por el tiempo depositacional (años $/ \mathrm{cm}$ ), dato calculado a partir de las edades interpoladas por el modelo de edad, posibilitando el cálculo de las tasas de acumulación de carbón (partículas/ $\mathrm{cm}^{3}$ /año). Los preparados palinológicos fueron depositados en el Laboratorio de Palinología y Ecología Vegetal del Campus Los Ángeles, de la Universidad de Concepción. Los porcentajes de los tipos polínicos fueron calculados sobre la suma total de la que se excluyeron las esporas de hongos y helechos y las micropartículas de carbón, para evitar su sobrerrepresentación en el perfil. Con ayuda del programa Coniss-TILIA (Grimm 1987), se construyó el diagrama polínico con un dendrograma de asociatividad florística. Se dispuso de muestras de sedimento para obtener fechados a distintos niveles en el testigo, los que 
fueron seleccionados en relación a los diferentes quiebres que presenta la curva polínica. Se realizó un fechado radiocarbónico en el laboratorio Beta Analytic Inc. (Florida, U.S.A.). La edad se expresa en edad calibrada $\mathrm{C}^{14}$ antes del presente (cal. años AP) (Tabla I), utilizando el software 5.0.1 CALIB (Stuiver et al. 2005) y calibrado con la curva de calibración del Hemisferio Sur (SHCal04) (McCormac et al. 2004). También se realizaron tres dataciones radioisotópicas de $\mathrm{PB}^{210}$ (Tabla II). Estos análisis fueron realizados en el servicio de radioisótopos de la Universidad de Sevilla, España. Los modelos de edad relacionados para cada profundidad se obtuvieron por el software MCAge mediante un spline cúbico suavizado y una aproximación de bootstrap (Muestreo de Monte Carlo) (Higuera 2009).

TABLA I. Datación radiocarbónica AMS realizada en el registro sedimentario de la turbera Chepu (*Mediana de probabilidad).

TABLE I. Radiocarbon $\left(\mathrm{C}^{14}\right)$ dating of the sedimentary record of the Chepu peat bog (*Median of probability).

\begin{tabular}{cccc} 
Código de la datación & Profundidad $(\mathrm{cm})$ & Años $\mathrm{C}^{14} \mathrm{AP}+/-1 \sigma$ & Años cal. AD \\
\hline Beta- 301134 & $17,5-20,0$ & $2020+/-30$ & $* 34$ \\
\hline
\end{tabular}

TABLA II. Dataciones realizadas con el método de $\mathrm{PB}^{210}$ en el registro sedimentario de la turbera Chepu.

TABLE II. Dating using the $\mathrm{PB}^{210}$ method of the sedimentary record of the Chepu peat bog.

\begin{tabular}{cccc}
\hline Código de la datación & Profundidad $(\mathrm{cm})$ & Error años PB PB $^{210}$ & Edad AÑos AD \\
\hline CH 1-1 & 3 & 3 & 1983 \\
CH 1-2 & 7 & 10 & 1961 \\
CH 1-3 & 13 & 1 & 1897 \\
\hline
\end{tabular}

\section{RESULTADOS}

El registro polínico del perfil Chepu (Fig. 2) presenta una diversidad de 27 taxa, con dominancia de Poaceae, Gunnera y Gaultheria. Con ayuda del programa Coniss-Tilia, se reconocen en el diagrama dos zonas polínicas, que de base a techo, se describen a continuación:

Zona CHP-I: $20 \mathrm{~cm}$ a 7,5 cm (368 años cal. AC y 1960 años cal. AD). La base del perfil muestra un porcentaje alto de abundancia de Poaceae (35-45\%) y Gunnera (89\%), seguido por los taxa arbustivos Gaultheria (5-10\%) y Empetrum (2-8\%). Hacia la parte media de esta zona polínica se destaca en abundancia relativa el polen de Weinmannia trichosperma Cav., taxón característico del bosque templado valdiviano (12\%), junto con la presencia de compuestas $(7 \%)$.

Las Poaceae (40-55\%) dominan ampliamente las abundancias relativas de los taxa representados en la zona. Hacia el techo de la misma, Gunnera (5-25\%) alcanza sus mayores porcentajes de abundancia relativa. Asteraceae (4$12 \%)$ y Chenopodiaceae (2-3\%) alcanzan las abundancias más importantes de su registro en el estrato no arbóreo, acompañando a Poaceae. Callitriche (2-3\%), Potamogeton (2-3\%) y Astelia pumila (G. Forst.) Gaudich. (1-3\%) aportan la condición de humedad en el ecosistema, a lo que se suma la abundancia relativa del briofito Sphagnum (4-5\%).
Zona CHP-II: $7,5 \mathrm{~cm}$ a $0 \mathrm{~cm}$ (1960-2010 años cal. AD). Esta zona representa los últimos $7,5 \mathrm{~cm}$ del perfil. La base de la secuencia se caracteriza por la máxima representación porcentual de Tepualia stipularis (14\%), Nothofagus tipo antarctica/dombeyi (4\%) y Eucryphia/ Caldcluvia (9\%), junto a un porcentaje de abundancia bajo de especies de Mirtáceas (2\%). Los taxa de helechos del tipo Hymenophyllum y Polypodium se presentan con trazas que sumados alcanzan el 3\% de participación en el registro. Hacia el techo de la zona, que se corresponde con el techo del perfil, se observa una diversidad de taxa polínicos correspondientes a un componente mayoritariamente arbustivo, donde destacan los géneros Gaultheria, Ugni, Empetrum y Misodendrum, acompañados por las herbáceas del tipo Poaceae, Asteraceae, Apiaceae, Rubiaceae. El componente palustre está representado por los taxa Juncaceae, Callitriche, Cyperaceae, Potamogeton y Astelia pumila que sumados alcanzan un $3 \%$ de representación en el registro, acompañados de una baja presencia del musgo Sphagnum.

Uno de los cambios más evidentes en el diagrama polínico es el que se observa respecto del comportamiento de Poaceae, con una marcada dominancia en la zona CHP-I y que posteriormente decrece hacia el techo de la misma zona, coincidiendo a esta misma profundidad con el incremento máximo del taxa palustre Gunnera (Fig. 2), lo que representa la transición entre una composición vegetal 
de tipo herbáceo, asociado a un ecosistema con estructura de pastizal abierto y el comienzo del pantano, con dominancia de un elemento vegetal colonizador característico del ambiente palustre, como es Gunnera, acompañado por la presencia de elementos polínicos asociados a condiciones de humedad tales como Juncaceae, Callitriche, Potamogeton, Astelia pumila y Sphagnum.

ANÁLISIS DE PARTíCULAS MACROSCÓPICAS DE CARBÓN

El análisis de la serie temporal de partículas macroscópicas de carbón de la turbera Chepu y los valores de la tasa de acumulación de carbón junto con la cronología del perfil se muestran en la Figura 3. La cronología utilizada fue elaborada sobre la base de las profundidades corregidas y edades años calendario.

En la turbera Chepu se presenta una marcada actividad de fuego aproximadamente en el año $1900 \mathrm{AD}(12 \mathrm{~cm})$, lo que se evidencia en una alta tasa de acumulación de partículas macroscópicas de carbón (Charcoal Influx). Para los años posteriores se presenta una tasa de acumulación más decreciente en el tiempo, pero constante, aproximadamente por 60 años (entre 11 y $5 \mathrm{~cm}$ ). Hacia el año $1972 \mathrm{AD}$ se puede identificar otro evento de fuego importante (entre 6 y $5 \mathrm{~cm})$.

\section{DISCUSIÓN}

El registro polínico de la turbera Chepu muestra un predominio de taxa característicos de un pastizal húmedo con Gunnera y Sphagnum, con presencia de elementos característicos del bosque valdiviano, en un contexto de permanente dominancia de Poaceae durante los últimos 2.000 años. No obstante esta dominancia, se pueden describir algunas fluctuaciones en el registro polínico. En el tramo basal del perfil (entre 20 y $15 \mathrm{~cm}$ ) (400 años cal AC-1.200 años cal AD), es posible observar un pastizal de Poaceae acompañado de Asteraceae, Gaultheria, Empetrum y Gunnera, que proporciona en su conjunto, una idea de un ambiente húmedo, probablemente desarrollado en suelo con mal drenaje, abierto y frío, que facilita la presencia de este matorral bajo asociado a Gunnera, un taxón pionero y colonizador, característico de ambientes húmedos (Pisano 1977, Rondanelli 2001, Fesq-Martin et al. 2004), el cual irá cobrando mayor significancia porcentual en el registro polínico de Chepu hasta alcanzar el techo de la zona CHP I. Sobre los $15 \mathrm{~cm}, \sim 1250$ años cal. AD, comienza a disminuir el matorral bajo asociado a Poaceae y es ésta quien presenta un incremento notorio de su abundancia relativa, alcanzando hacia el año $1900 \mathrm{AD}$, más del $50 \%$ de su representación polínica en el perfil Chepu, caracterizando botánicamente la zona CHP I. Asteraceae y Gunnera acompañan este dominio de las gramíneas, pero con porcentajes menores que, no obstante, le permiten mantener la condición de humedad del ecosistema, al cual se añade progresivamente el componente de las palustres, entre ellas el desarrollo de Sphagnum y Callitriche. En este contexto, el componente arbóreo para CHP I está representado principalmente por Weinmannia, seguido de Tepualia y Eucryphia/Caldcluvia, elementos del bosque valdiviano, que si bien aún no alcanzan sus máximas frecuencias de abundancias en el perfil, se ajustan bien a esta propuesta edafoclimática local de una condición más bien húmeda y con drenaje deficiente del suelo (Veblen \& Schlegel 1982, Weinberger 1997). El establecimiento de esta composición florística en Chepu, que abarca gran parte de la zona polínica CHP I, transcurre entre el año 1250 y el año $1900 \mathrm{AD}$, aproximadamente, período para el cual se ha sugerido un aumento de las precipitaciones para la región de Chiloé (Abarzúa 2004) y para la Región de Los Lagos (Bertrand et al. 2005), y un descenso en el promedio de las temperaturas de verano para toda esta región (Lara \& Villalba 1993), lo que refuerza la idea de una condición climática húmeda y más bien fría para CHP I.

A partir del año $1900 \mathrm{AD}$, aproximadamente, podemos hacer una interpretación conjunta entre el diagrama polínico (Fig. 2) y el diagrama de las tasas de acumulación de macrocarbón (Fig. 3), en Chepu. En este último, observamos por primera vez un incremento en la curva de las tasas de acumulación de partículas macroscópicas, notorio respecto de los años anteriores, que asociado al efecto local de fuego (incendio) podría explicar la disminución evidente, por ejemplo, del componente arbóreo del perfil, interpretado como la consecuencia lógica de la pérdida de biomasa por acción del fuego, y al mismo tiempo, explicaría el posterior incremento en las concentraciones relativas de las especies, que tradicionalmente han sido citadas en la literatura como especies oportunistas, que aprovechan rápidamente el nicho disponible para aumentar su población, ejemplo de esto lo constituyen las Poaceae (Abarzúa 2009, Urrutia et al. 2010), Gunnera (Heusser 1995, Rondanelli 2001, FesqMartin et al. 2004) y Sphagnum (Díaz \& Armesto 2007, Armesto et al. 2009). Así, junto al efecto mancomunado del fuego, en un ambiente con prevalencia de mayores aportes hídricos, se desarrolló la conformación del pastizal húmedo de gramíneas, nalcas y musgos, con ausencia del elemento boscoso, que caracteriza a esta zona polínica. Esto, hasta el año $1960 \mathrm{AD}$, donde por efecto de la geotectónica, Chile centro-sur se ve enfrentado al terremoto más grande de su historia, con el consecuente tsunami que alteró drásticamente la geografía litoral del país, entre Valdivia y el norte de la Isla Grande de Chiloé (Cisternas et al. 2000, 2005, Lagos \& Cisternas 2004). El perfil polínico de Chepu evidencia este cambio. La subducción de la plataforma continental litoral producto del megaterremoto, con el consecuente ingreso de agua oceánica al ecosistema palustre, generó un ambiente deltaico mixto que afectó a la comunidad vegetal hidrófila 


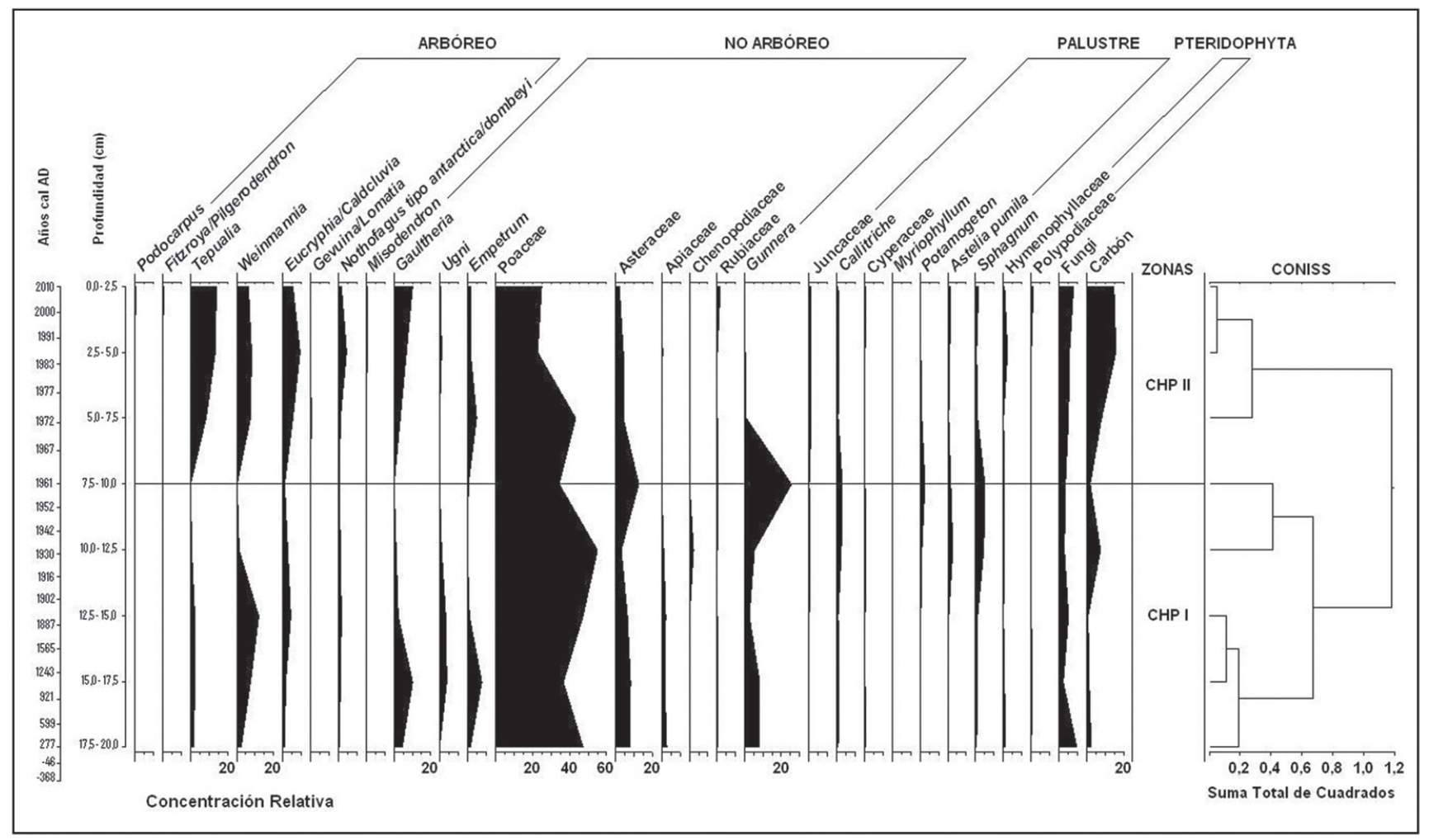

Figura 2. Diagrama de polen y de micropartículas de carbón, en porcentajes, de Turbera Chepu (4203'10,8” S; 7359’56,8” W), Isla Grande de Chiloé, Chile.

FIGURE 2. Diagram of pollen and charcoal microparticles, in relative concentration, of the Chepu peat bog (4203'10.8" S; 7359'56.8' W), Great Island of Chiloé, Chile.

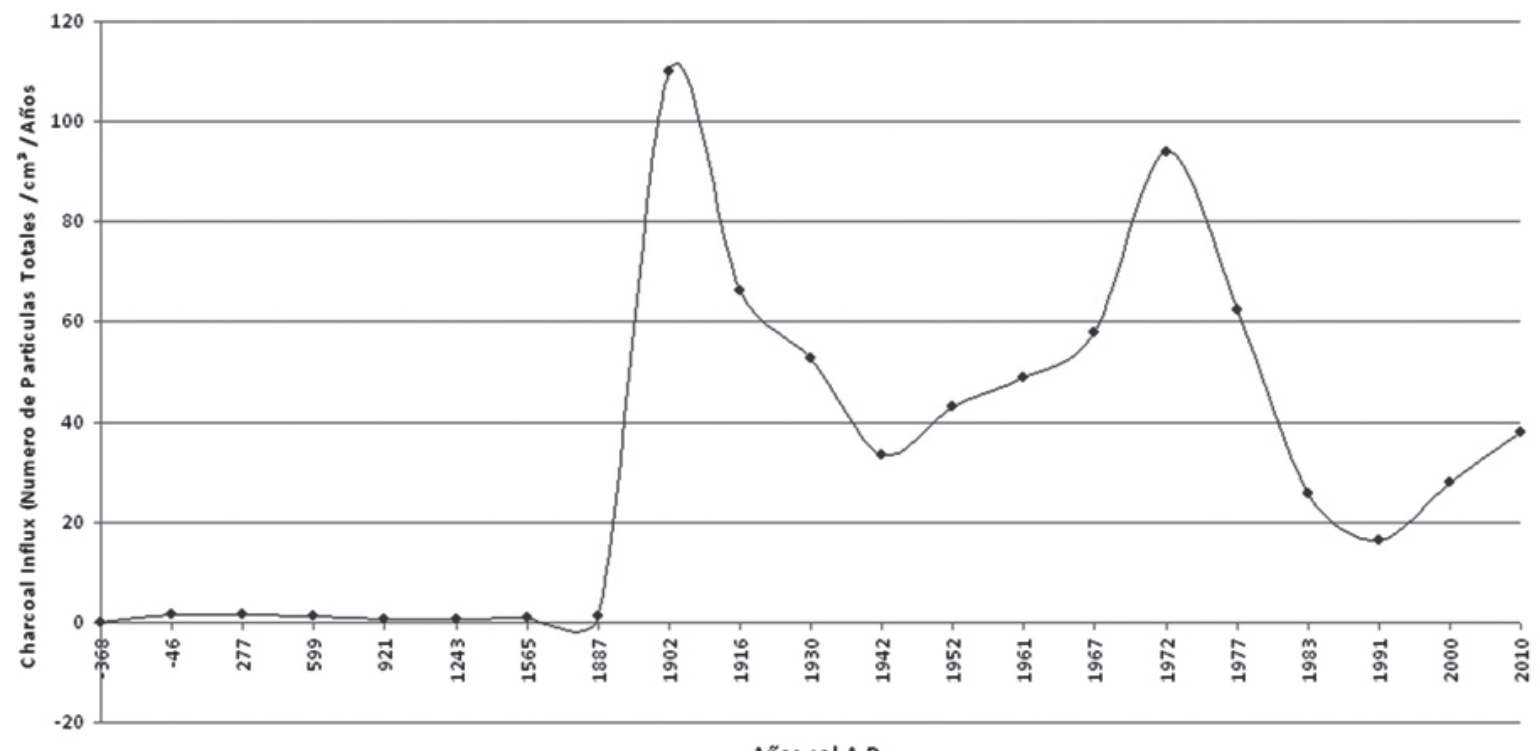

Años cal A.D.

Figura 3. Tasas de acumulación de partículas macroscópicas (Charcoal Influx) de carbón de la turbera Chepu (TA $=\mathrm{N}^{\mathrm{o}}$ partículas totales/ $\mathrm{cm}^{3} /$ año). Las edades se presentan en años calendarios AD (Anno Domini).

FigURE 3. Macroscopic particle accumulation rates (Charcoal Influx) of charcoal in the Chepu peat bog (TA = Total number of particles/ $\mathrm{cm}^{3} /$ year). Ages are presented in $\mathrm{AD}$ (Anno Domini) calendar years. 
presente; disminuyen prácticamente todos los taxa del perfil y aunque se aprecia una recuperación del ecosistema, más o menos rápido en cuanto a tiempo, la composición del pastizal no vuelve a ser la caracterizada para la zona polínica CHP I. Esta situación nos permite describir a partir de este evento, lo que denominamos la zona polínica CHP II del perfil, que se proyecta hasta el techo de la sección. Por las abundancias relativas registradas, del componente palustre y no-arbóreo de CHP II, podemos inferir una probable disminución en las condiciones de humedad del sitio, respecto de CHP I. El componente palustre se presenta muy restringido; disminuye la abundancia de Sphagnum, Callitriche y Potamogeton, éstas últimas solo se registran como trazas en los centímetros finales del perfil y Gunnera decrece hasta desaparecer del registro. Si bien, Poaceae sigue siendo dominante en el perfil, en esta zona decrece su abundancia relativa y comparte la composición florística con más elementos del matorral y sobre todo, del estrato arbóreo. En este último se aprecia un aumento progresivo de Tepualia, Weinmannia y Eucryphia/ Caldcluvia, las que alcanzan sus abundancias polínicas máximas en el registro, respondiendo, probablemente, a un cambio ambiental del ecosistema hacia una condición más termófila; situación semejante ha sido descrita ya para Chiloé (Villagrán 2001). El aporte de Nothofagus es más bien discreto y correspondería a un transporte a distancia desde ecosistemas interiores de mayor elevación.

$\mathrm{Al}$ analizar conjuntamente esta zona polínica con el diagrama de las tasas de acumulación de macrocarbón (Fig. 3 ), podemos observar que existe un segundo incremento notorio en la curva de las tasas de acumulación de partículas macroscópicas, para el año 1972, que sugeriría un segundo evento de fuego en el ecosistema, pero esta vez unido a una condición edafoclimática bastante menos húmeda, con un terreno mejor drenado, evidenciado por el tipo de vegetación descrita para esta zona polínica de Chepu II, que favoreció la regeneración del componente matorral-arbóreo por sobre el palustre. Por ejemplo, estudios realizados en Weinmannia trichosperma señalan que al generarse apertura del dosel en una escala mayor, como cuando ocurren incendios forestales, se favorece el incremento de esta especie pionera (Veblen 1985, Donoso et al. 1993, Armesto et al. 1996). Es interesante hacer notar que para ambos eventos de incendios sugeridos en el perfil de Chepu (1900 AD y 1972 AD), existe concordancia cronológica entre los máximos incrementos alcanzados por la curva de acumulación de partículas de macrocarbón (Fig. 3) y los incrementos máximos relativos observados de la curva de micropartículas de carbón registrados en el perfil polínico (Fig. 2); una concordancia entre fuego local y extralocal para la región.

Si bien el análisis de las macropartículas de carbón nos permite hablar de fuegos locales (Whitlock \& Larsen 2001, Whitlock et al. 2007), resulta más complejo poder relacionarlos con la causa de los mismos. La evidencia arqueológica de la zona de estudio da cuenta de la presencia de humanos desde hace varios miles de años, pero no permite tener una referencia clara de la influencia que las culturas originarias pudieron ejercer sobre la vegetación y los regímenes de fuego (Torrejón et al. 2011). Registros históricos para los últimos 400 años evidencian la influencia que ejerció la colonización española en la región, a través del despeje de áreas prístinas para el asentamiento humano (Weber 1903, Oyarzún 1934, Trivero 2005, Mancilla \& Rehbein 2007) y para tierras destinadas a los cultivos y ganadería, a consecuencia de la expansión progresiva de la población (Gómez de Vidaurre 1889, Elizalde 1970, Armesto et al. 1994). De estos antecedentes podríamos inferir que los incendios registrados en el influjo de las macropartículas de carbón (Fig. 3), indicados para el año $1900 \mathrm{AD}$ y para el año $1972 \mathrm{AD}$, aproximadamente, corresponderían muy probablemente a eventos relacionados con las actividades antrópicas señaladas precedentemente. Hacia finales del siglo XIX y comienzos del XX hubo intensos y extensos incendios forestales registrados en la zona sur de Chile, incluyendo la Isla Grande de Chiloé, algunos de los cuales han sido documentados como "incendios fuera de control" (Quintanilla 2004). Igualmente, podemos inferir que el evento de comienzos de los años 70 pudo estar ligado a la actividad forestal que comenzaba en el país, a través de la introducción intensiva del pino insigne, para lo cual hubo que despejar áreas ocupadas por la vegetación nativa (Quintanilla 2005).

Los datos obtenidos en este estudio son aún insuficientes como para determinar categóricamente una influencia climática de tipo zonal para el sector estudiado. No obstante, el tipo de ambiente para la zona polínica CHP I, descrito como de alta humedad producto de un aumento en las precipitaciones locales, en conjunto con la interpolación de los años calendarios, específicamente para el tramo entre el año $1250 \mathrm{AD}$ y el año $1800 \mathrm{AD}$, coincidiría con el período denominado Pequeña Edad de Hielo, cuya influencia climática ha sido informada como operante para el extremo sur de Sudamérica (Villalba et al. 2003, Urrutia et al. 2010). Serían necesarios nuevos registros, de mayor resolución temporal y espacial en el sector de estudio, para poder elaborar alguna hipótesis más fundamentada, relacionada con este tema.

Finalmente, nos parece necesario destacar el trabajo de Godley \& Moar (1973) en las turberas de Chiloé, donde uno de los testigos estudiados palinológicamente provino de la misma turbera analizada en la presente investigación, la turbera de origen antrópico de Chepu. Al comparar ambos perfiles polínicos, respetando la coincidencia aproximada de sus respectivas profundidades, se puede observar que existe similitud respecto de la diversidad de taxa, como de las abundancias relativas de ellas, de esta forma la interpretación de Godley \& Moar (1973), para 
los centímetros finales de su perfil, se condice con nuestra interpretación general para el perfil Chepu, describiéndolo como un ecosistema en donde se observa un predominio de taxa característicos de un pastizal húmedo, con Gunnera y Sphagnum, y con presencia de elementos característicos del bosque valdiviano. Lamentablemente, dicho trabajo carece de dataciones geocronológicas, por lo que no es posible aproximar la variable del tiempo calendario a la secuencia polínica observada.

\section{CONCLUSIONES}

La turbera Chepu, ubicada en el límite sur del bosque Valdiviano, en la Isla Grande de Chiloé, permite reconstruir una historia polínica y de historia de fuegos para los últimos 2000 años y su relación con las condiciones locales del clima y efectos antrópicos. Su historia vegetal está caracterizada por la presencia recurrente del pastizal húmedo de Poaceae, Gunnera y Sphagnum que ha transitado desde una condición de ecosistema abierto, frío y húmedo, entre aproximadamente el año $1250 \mathrm{AD}$ y el año $1800 \mathrm{AD}$, a uno de carácter más cerrado, de menor humedad y más termófilo, donde comparte presencia con elementos del bosque tipo valdiviano tales como Tepualia, Weinmannia y Eucryphia/Caldcluvia. Este último ambiente se consolida hasta nuestros días a partir de 1960, post acción del megaterremoto del mismo año, que afectó a gran parte de Chile centro-sur y cuya acción también es posible inferir a partir del registro polínico de Chepu, en donde para esa fecha se registra la disminución prácticamente total de todas las abundancias relativas de los taxa vegetales. El registro de las macropartículas de carbón para el perfil Chepu indicaría actividad de fuego, asociada a incendios forestales, solo para el siglo recién pasado, aproximadamente en el año $1900 \mathrm{AD}$ y en el año $1972 \mathrm{AD}$, los cuales han sido asignados como consecuencia probable de la expansión de terrenos para asentamiento poblacional, a inicios del siglo XX y como práctica silvícola, para favorecer las plantaciones forestales con especies introducidas, respectivamente.

\section{AGRADECIMIENTOS}

JMTC agradece al Dr. Gonzalo Sottile y colegas del laboratorio de Paleoecología y Palinología de la Universidad Nacional de Mar del Plata y al Proyecto U. Complutense de Madrid (UCM 4138114) con el apoyo de las Dras. Carolina León y Gisela Oliván. MJRR agradece a la Vicerrectoría Académica de la Universidad de Concepción por el permiso Sabático otorgado en 2013, que permitió, entre otras, el desarrollo de la presente investigación.

\section{BIBLIOGRAFÍA}

Abarzúa, A.M. 2004. Historia deglacial y postglacial de los bosques templado-lluviosos en la Isla Grande de Chiloé, Chile. Tesis Magíster en Ciencias Biológicas. Facultad de Ciencias, Universidad de Chile. Santiago, Chile. 65 pp.

AbarzÚA, A.M. 2009. Respuestas ambientales a cambios climáticos y culturales en la Región de la Araucanía, Chile. Tesis. Doctorado en Ciencias Forestales. Facultad de Ciencias Forestales, Universidad Austral de Chile. Valdivia, Chile. $74 \mathrm{pp}$.

AbarzúA, A.M. \& P.I. Moreno. 2008. Changing fire regimes in the temperate rainforest region of southern Chile over the last 16,000 yr. Quaternary Research 69: 62-71.

Abarzúa, A.M., C. Villagrán \& P.I. Moreno. 2004. Deglacial and postglacial climate history in east-central Isla Grande de Chiloé, southern Chile $\left(43^{\circ} \mathrm{S}\right)$. Quaternary Research 62: 49-59.

Armesto, J., C. Villagrán \& C. Donoso. 1994. Desde la era glacial a la industrial: la historia del bosque templado chileno. Ambiente y Desarrollo 10: 66-72.

Armesto, J., J.C. Aravena, C. Villagrán, C. Pérez \& G. Parker. 1996. Bosques templados de la Cordillera de la Costa. En: J. Armesto, C. Villagrán \& M.T.K. Arroyo (eds.), Ecología de los bosques nativos de Chile, pp. 199-213. Editorial Universitaria, Santiago, Chile.

Armesto, J., M. Bustamante-Sánchez, M.F. Díaz, M. González, A. Holtz, M. NúÑez-Avila \& C. Smith-Ramírez. 2009. Fire disturbance regimes, ecosystem recovery and restoration strategies in Mediterranean and temperate regions of Chile. In: A. Cerda \& P.R. Robuchaud (eds.), Fire effects on soils and restoration strategies, pp. 537-567. Science Publishers, New Hampshire, USA.

Bertrand, S., X. Boës, J. Castiaux, F. Charlet, R. Urrutia, C. Espinoza, B. Charlier, G. Lepoint \& N. Fagel. 2005. Temporal evolution of sediment supply in Puyehue Lake (Southern Chile) during the last 600 years: climatic significance. Quaternary Research 64: 163-175.

Bianchi, M., \& H. D’Antoni. 1986. Depositación del polen actual en los alrededores de Sierra de Los Padres (Pcia. de Buenos Aires). En: IV Congreso Argentino de Paleontología y Bioestratigrafía, Apéndice de las Actas, pp. 16-27. Mendoza, Argentina.

Carmona, M.R., J.C. Aravena, M.A. Bustamante-Sánchez, J.L. Celis-Diez \& A. Charrier. 2010. Estación biológica Senda Darwin: investigación ecológica de largo plazo en la interfase ciencia-sociedad. Revista Chilena de Historia Natural 83: 113-142.

Cisternas, M., I. Contreras \& A. Araneda. 2000. Reconocimiento y caracterización de la facies sedimentaria depositada por el tsunami de 1960 en el estuario Maullín, Chile. Revista Geológica de Chile 27(1): 3-11.

Cisternas, M., B. Atwater, F. Torrejón, Y. Sawai, G. Machuca, M. Lagos, A. Eipert, C. Youlton, I. Salgado, T. Kamataki, M. Shishikura, C.P. Rajendran, J. Malik, Y. Rizal \& M. Husni. 2005. Predecessors of the giant 1960 Chile earthquake. Nature 437: 404-407.

Di Castri, F. \& E. Hajek. 1976. Bioclimatología de Chile. Vicerrectoría Académica Universidad Católica, Santiago. 129 pp. 
DíAz, M.F. 2004. Limitantes biológicas e hidrológicas de la sucesión secundaria en bosques de Chiloé. Tesis. Doctorado en Ciencias. Facultad de Ciencias, Universidad de Chile. Santiago, Chile. 164 pp.

Díaz, M. \& J. Armesto. 2007. Limitantes físicos y bióticos de la regeneración arbórea en matorrales sucesionales de la Isla Grande de Chiloé, Chile. Revista Chilena de Historia Natural 80: 13-26.

Donoso, C., R. Grez, B. Escobar \& P. Real. 1984. Estructura y dinámica de bosques del tipo forestal siempreverde en el sector de Chiloé insular. Bosque 5(2): 82-104.

Donoso, C., V. Sandoval, R. Grez \& J. Rodríguez. 1993. Dynamics of Fitzroya cupressoides forests in southern Chile. Journal of Vegetation Science 4: 303-312.

Echeverría, C., A.C. Newton, A. Lara, J.M. Benayas \& D.A. Coomes. 2007. Impacts of forest fragmentation on species composition and forest structure in the temperate landscape of southern Chile. Global Ecology and Biogeography 16: 426-439.

Elizalde, R. 1970. La sobrevivencia de Chile. Ministerio de Agricultura, Servicio Agrícola y Ganadero, Santiago. 492 pp.

Faegri, K. \& J. Iversen.1989. Textbook of Pollen Analysis. John Wiley \& Sons, New York. 328 pp.

Fesq-Martin, M., A. Friedmann, M. Peters, J. Behrmann \& R. KiLIAN. 2004. Late-glacial and Holocene vegetation history of the Magellanic rain forest in southwestern Patagonia, Chile. Vegetation History \& Archaeobotany 13: 249-255.

Godley, E.J. \& N.T. Moar. 1973. Vegetation and pollen of two bogs on Chiloé. New Zealand Journal of Botany 11: 25568.

Gómez de Vidaurre, F. 1889. Historia Geográfica, Natural y Civil del Reino de Chile en 1748. Imprenta Ercilla, Santiago de Chile. 357 pp.

Grimm, E. 1987. Coniss: A fortran 77 Program for stratigraphically constrained Cluster analysis by the method of incremental sum of squares. Computers Geoscience 13: 13-35.

Heusser, C. 1987. Fire history of Fuego-Patagonia. Quaternary of South America and Antarctic Peninsula 5: 93-109.

Heusser, C. 1995. Three Late Quaternary pollen diagrams from southern Patagonia and their paleoecological implications. Palaeogeography, Palaeoclimatology, Palaeoecology 118: $1-24$.

Higuera, P.E. 2009. CharAnalysis. University of Idaho, Moscow, Idaho. [software and manual] Accessed on May 5, 2009 at URL:http://sites.google.com/site/charanalysis.

Huber, U.M., V. Markgraf \& F. Schäbitz. 2004. Geographical and temporal trends in Late Quaternary fire histories of Fuego-Patagonia. South America Quaternary Science Reviews 23: 191-198.

IturRaspe, R. \& C.E. Roig. 2000. Aspectos hidrológicos de turberas de Sphagnum de Tierra del Fuego -Argentina. En: A. Coronato \& C. Roig (eds.), Conservación de ecosistemas a nivel mundial con énfasis en las turberas de Tierra del Fuego. Disertaciones y Conclusiones, pp. 85-93. Ushuaia, Argentina.

Jaña-Prado, R.C., J.L. Celis-Diez, A.G. Gutiérrez, C. Cornelius \& J.J. Armesto. 2006. Diversidad en bosques fragmentados de Chiloé: ¿Son todos los fragmentos iguales? En: A. Grez, J. Simonetti \& R. Bustamante (eds.), Biodiversidad en ambientes fragmentados de Chile: Patrones y procesos a diferentes escalas, pp. 159-190. Editorial Universitaria, Santiago, Chile.

Lagos, M. \& M. Cisternas. 2004. Tsunami deposits as risk indicators: sedimentary evidences. Revista Geográfica de Chile Terra Australis 49: 329-351.

LARA, A. \& R. Villalba. 1993. A 3,620-year temperature reconstruction from Fitzroya cupressoides tree rings in southern South America. Science 260: 1104-1106.

Markgraf, V. 1989. Paleoclimates in central and South America since 18,000 BP based on pollen and lake-level records. Quaternary Science Reviews 8: 1-24.

Markgraf, V., P. Bradbury, A. Schwalb, S. Burns, Ch. Stern, D. Arizategui, A. Gilli, F. Anselmetti, S. Stine \& N. MaidanA. 2003. Holocene palaeoclimates of southern Patagonia: limnological and environmental history of Lago Cardiel, Argentina. The Holocene 13: 581-591.

Mancilla, M. \& M. Rehbein. 2007. De viajes a retornos: Una aproximación al estudio del imaginario de la vida errante en el Chiloé de la primera mitad del siglo XX. Tesis. Antropología. Facultad de Filosofía y Humanidades, Universidad Austral de Chile, Valdivia, Chile. 183 pp.

McCormac, F.G., A.G. Hogg, P.G. Blackwell, C.E. Buck, T.F. Higham \& P.J. Reimer. 2004. SHCal04 Southern Hemisphere Calibration 0 - 1000 cal BP. Radiocarbon 46: 1087-1092.

Moreno, P.I., M.R. Kaplan, J.P. François, R. Villa-Martínez, C.M. Moy, C.R. Stern \& P.W. Kubik. 2009a. Renewed glacial activity during the Antarctic Cold Reversal and persistence of cold conditions until $11.5 \mathrm{ka}$ in SW Patagonia. Geology 37: 375-378.

Moreno, P.I., J.P. François, R. Villa-Martínez \& C.M. Moy. 2009b. Millennial-scale variability in Southern Hemisphere westerly wind activity over the last 5000 years in SW Patagonia. Quaternary Science Reviews 28: 25-38.

Moreno, P.I., T. Kitzberger, V. Iglesias \& A. Holz. 2010. Paleofires in southern South America since the Last Glacial Maximum. Pages News 18: 75-77.

Moreno, P.I., R. Villa-Martínez, M.L. Cárdenas \& E.A. Sagredo. 2012. Deglacial changes of the southern margin of the southern westerly winds revealed by terrestrial records from SW Patagonia $\left(52^{\circ} \mathrm{S}\right)$. Quaternary Science Reviews 41:1-21.

Oyarzún, A. 1934. Cultura aborigen de Chiloé. Revista Chilena de Historia y Geografía 83: 235-254.

Pisano, E. 1977. Fitogeografía de Fuego-Patagonia Chilena. I. Comunidades vegetales entre las latitudes $52^{\circ}$ y $56^{\circ} \mathrm{S}$. Anales del Instituto de la Patagonia 8: 121-250.

Quintanilla, V. 2004. Degradación del bosque pluvial en una cuenca hidrográfica del norte de la Isla Grande de Chiloé. Revista de Geografía Norte Grande 31: 73-84.

Quintanilla, V. 2005. Estado de recuperación del bosque nativo en una cuenca nordpatagónica de Chile, perturbada por grandes fuegos acaecidos 50 años atrás $\left(44^{\circ}-45^{\circ} \mathrm{S}\right)$. Revista de Geografía Norte Grande 39: 73-92.

Rondanelli, M. 2001. Historia de la vegetación andina en los valles de Alto Biobío y Lonquimay, Chile centro-sur $\left(38^{\circ}-39^{\circ} \mathrm{S}\right)$, durante el Holoceno. Estudio paleoecológico basado en el análisis de polen. Tesis. Doctorado en Ciencias Biológicas. Facultad de Ciencias Naturales y 
Oceanográficas, Universidad de Concepción, Concepción, Chile. 93 pp.

Rondanelli, M., J. Max Troncoso \& C. León. 2011. Historia Vegetal Reciente en Patagonia Occidental. Análisis

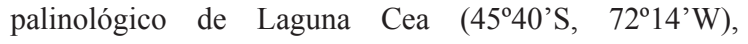
Coyhaique, Chile. Revista Polibotánica 32: 157-172.

Schlatter, R. \& J. Schlatter. 2004. Los turbales de Chile. En: D. Blanco \& V. de la Balze (eds.), Los Turbales en Patagonia. Bases para su inventario y la conservación. Cap. 5, pp. 7580. Wetlands International, Buenos Aires, Argentina.

Stuiver, M., P.J. Reimer, \& R.W. Reimer. 2005. CALIB 5.0.2. http://calib.qub.ac.uk/calib/.

Teneb, E. \& O. Dollenz. 2004. Distribución espacial de la flora vascular, humedad y $\mathrm{pH}$ en un turbal de esfagno (Sphagnum magellanicum Brid.), Magallanes, Chile. Anales Instituto de la Patagonia 32: 5-12.

Torrejón, F., M. Cisternas, I. Alvial \& L. Torres. 2011. Consecuencias de la tala maderera colonial en los bosques de alerce de Chiloé, sur de Chile (siglos XVI-XIX). Magallania 39: 75-95.

Trivero, A. 2005. Los primeros pobladores de Chiloé. Génesis del horizonte mapuche. Ñuke Mapuförlaget, URL: http://mapuche.info.scorpionshops.com/mapuint/ Trivero050900.pdf Visitado: 20 de abril, 2014.

Urrutia, R., A. Araneda, L. Torres, F. Cruces, C. Vivero, F. Torrejón, R. Barra, N. Fagel \& B. Scharf. 2010. Late Holocene environmental changes inferred from diatom, chironomid, and pollen assemblages in an Andean lake in Central Chile, Lake Laja ( $\left.36^{\circ} \mathrm{S}\right)$ Hydrobiologia 648 (1): 207-225.

Veblen, T. \& F. Schlegel. 1982. Reseña ecológica de los bosques del sur de Chile. Bosque, 4(2):73-115.

VeBlen, T.T. 1985. Forest development in tree-fall gaps in the temperate rain forests of Chile. National Geographic Research 1: 161-184.

Villagrán, C. 2001. A model for the history of vegetation of the Coastal Range of central southern Chile: Darwin's glacial hypothesis. Revista Chilena de Historia Natural 74: 793803.
Villagrán, C., P. Moreno \& R. Villa. 1996. Antecedentes palinológicos acerca de la historia cuaternaria de los bosques chilenos. En: J. Armesto, C. Villagrán \& M. Kalin (eds.), Ecología de los bosques nativos de Chile, pp. 51-69. Editorial Universitaria, Santiago, Chile.

Villalba, R., A. Lara, J. Boninsegna, M. Masiokas, S. Delgado, J. Aravena, F. Roig, A. Shmelter, A. Wolodarski \& A. RIPALTA. 2003. Large-scale temperature changes across the southern Andes: 20Th-century variations in the context of the past 400 years, Climatic change 59 (1-2): 177-232.

Weber, A. 1903. Chiloé su estado actual, su colonización, su porvenir. Imprenta Mejía, Santiago. $195 \mathrm{pp}$.

Weinberger, P. 1997. Definición de grupos ecológicos en formaciones boscosas siempreverdes de la zona austral de Chile. Revista Bosque 18(2): 29-41.

Whitlock, C. \& C.P.S. Larsen. 2001. Charcoal as a Fire Proxy. In J.P. Smol, H.J.B. Birks \& W.M. Last (eds.), Tracking Environmental Change Using Lake Sediments, Vol. 3 Terrestrial, Algal, and Siliceous indicators, pp. 75-97. Kluwer Academic Publishers, Dordrecht, The Netherlands.

Whitlock, C., P.I. Moreno \& P. Bartlein. 2007. Climatic controls of Holocene fire patterns in southern South America. Quaternary Research 68: 28-36.

Wille, M., N. Maidana, F. Schäbitz, M. Fey, T. Haberzettl, S. Janssen, A. Lücke, C. Mayrs, C. Ohlendorf, G. Schleser \& B. ZolitschKA. 2007. Vegetation and Climate dynamics in Southern South America: the microfossil record of Laguna Potrok Aike, Santa Cruz, Argentina. Reviews in Paleobotany and Palynolinology 146: 234-246.

Wille, M. \& F. ScHÄBITZ. 2009. Late-glacial and Holocene climate dynamics at the steppe/forest ecotone in southernmost Patagonia, Argentina: the pollen record from a fen near Brazo Sur, Lago Argentino. Vegetation History and Archaeobotany 18: 225- 234.

Zegers, G., J. Larraín, F. Díaz \& J. Armesto. 2006. Impacto ecológico y social de la explotación de pomponales y turberas de Sphagnum en la Isla Grande de Chiloé. Revista Ambiente y Desarrollo 22(1): 28-34.

Recibido: 20.05 .14

Aceptado: 10.09.15 